\title{
Cutting Through the Noise: Revamping Current Awareness at Irwin Mitchell
}

\begin{abstract}
This article by Jill Henderson and Louise Young is based on their 'Lightning Talk' at the 2018 BIALL Conference and describes the recent current awareness project at Irwin Mitchell. It outlines each stage of the project from recognising there was a need to change and choosing the right product to enable that, through to gathering requirements, building searches and launching the new alerts. It goes through each stage in detail and outlines the lessons learnt to help others who may be considering a similar project.
\end{abstract}

Keywords: law firms; legal information; current awareness

\section{INTRODUCTION}

Keeping on top of everything we need to be aware of is a challenge for all of us in an environment of increasing information overload. For knowledge and information professionals the challenge is not just how do we stay current ourselves, it's also how do we help our users to stay current on what matters to them.

That is not a simple problem to solve because there are so many variables to consider. Beyond just the sheer amount of information available and the reliability of that information you have to remember that every user is different and has different requirements in terms of what information they are interested in and how they want it delivered so there is never a one-size-fits-all approach.

This article will outline how the Knowledge \& Intelligence team at Irwin Mitchell used Manzama to help our lawyers and other users to cut through that noise.

\section{BACKGROUND}

Prior to this project current awareness at Irwin Mitchell would best be categorised as inconsistent. The Knowledge \& Intelligence team did offer bulletins, tracking and alerts as a service but this was done on a by request basis meaning only some teams received central updates, whilst others relied on individuals setting up their own alerts from various sources. The main reason for this was that the tools we used to create bulletins required a lot of manual curation and with such a small team the time taken to set up and produce these was prohibitive.

There were several consequences to this. Usually the bulletins and alerts that were requested or set up within teams focused purely on legal updates meaning whole swathes of the business were not receiving any sort of market intelligence updates. Everything was delivered by email with people getting the same information from multiple sources and all that duplication often resulted in it all just becoming 'white noise' meaning there was an increased chance of key legal changes being missed. There was also a tendency for teams to rely on a single person within a team to be 'up to date' and for people to not take personal responsibility for their own learning and development.

\section{HOW DID WE DO IT?}

Our starting point for this project was to identify what we really needed. It wasn't about getting the newest shiniest product but making sure that what we did select would support our team, our lawyers and their clients in cutting through the noise of current awareness and really allowing us to focus in on what they needed to know, when they needed to know it. Some of our key requirements for the product we wanted were:

- Automation with minimal duplication of content

- Easy customisation for our users

- The ability for our central Knowledge team to set up more complex searches where required.

- A full range of sources from all of our key providers with the ability to add additional sources ourselves as required.

To ensure we selected the right product we had meetings and demos with a number of different providers and decided that Manzama appeared to be the best fit for our requirements. We then ran a small two week trial with a group of interested people from different business areas. Based on this feedback we chose Manzama as our preferred supplier and started to plan out how we were going to manage the project.

There are two Knowledge \& Intelligence Advisors in our team at Irwin Mitchell, each of whom has 
responsibility for a specific part of the business. As the initial phase of the rollout was to focus on our lawyer population, each advisor was responsible for deploying this to their respective populations. The first part of the plan was for us to undertake some detailed training with the specialist team from Manzama so we could better understand how to roll this out to such a large number of people in the most effective way. We worked closely with Manzama on this as it was important to learn what had and hadn't been successful previously and ensure that we were using the product in the right way for the rollout to our particular groups of users across the business.

As there were over I,500 people to roll this out to initially, we decided that a phased approach would be best, starting with those teams and individuals who were currently receiving an update from us.

Phase one consisted of building the existing updates that we distributed into Manzama. There was a period of testing these in partnership with our users to check that the new updates contained all the key information they were currently receiving plus any additional requirements they flagged to us during the feedback cycle. This was very much a trial and error process and informed our approach for the following phases.

Moving into phase two, we used the feedback gathered previously and devised a simple survey using DotMailer which was designed to find out what type of information users would like to receive updates about and which sources they felt were important. We designed a survey for each practice area so that we could give examples of subject specific topics, industries, companies etc and gave some guidance as to what each section could consist of. So for example in the personal injury teams we knew that there were a number of key blogs that were widely subscribed to that we could include like the Civil Litigation Brief, and topics such as the discount rate, or medical negligence. We also provided a 'menu' of possibilities for people to choose from in each area. We then sent this out to key individuals within our practice teams who we identified might be interested in current awareness. This was largely people who made a lot of article requests, were receiving automated alerts from Lawtel, Lexis etc or other key contacts that we had within each of our teams.

We then used all of the survey responses to create template emails for each team containing the core content that was flagged as important, split into the six categories that Manzama uses (practices, industries, companies, competitors, topics and sources). We made sure that we included our journal content in the sources section to replace our existing table of contents delivery service that was done manually in our team. When we were ready to deploy these updates we used a service that Manzama provides called 'jump start'. This meant that their team set up all of the accounts that we needed and allocated the relevant content to each individual profile. This involved us setting up a group profile for each team and telling them which individuals needed to receive the content we had allocated to each group. This worked well as it took all of the account setup admin away from us but enabled us to be very targeted in our approach. There are some individuals who wanted content from across two groups (so a product liability lawyer might want wider medical negligence content too, or a partner might need real estate news but also law firm management articles), and using this approach made that possible.

The final phase was to roll this out to the rest of the business, but as we had done the above ground work and had the templates and groups in place, that was relatively pain free. We did this over a number of months using the jump start process again on a team by team basis. We were still getting lots of feedback at this stage on content and continually working on this as it was such a crucial part of the success of this project.

\section{GETTING THE CONTENT RIGHT FOR US}

One of the main drivers behind this project was to ensure that all current awareness being sent and received by our lawyers was bespoke to them, so a big part of this project was the creation of the searches that would provide this content.

Manzama has 1000s of pre-configured searches called tokens for all of its categories of content along with a comprehensive source directory. Some of these tokens were suitable for our needs but others needed to be refined in order to deliver content useful to our lawyers. A lot of this was done by using the existing filters built into the system - the most popular were the geographical filters and also the lists of 'top sources' which then excluded a lot of the less relevant content.

Alongside this we created over 300 unique searches on niche areas that Manzama didn't already cover and added in lots of key sources and source lists to run the searches over. A good example of this is a number of searches we set up for our personal injury lawyers as there weren't relevant tokens for a lot of the niche work we do in this area. The ability to add in sources manually via RSS was invaluable here, as we were able to set up searches on our key legal research databases and then add these in as RSS feeds. This meant that as well as being able to deliver the sector news, and track key companies and competitors, we were able to bring together content from Lexis and Thomson Reuters in one search feed to deliver really bespoke legal updates from a truly complete set of sources. We were also able to 'boost' specific sources to some groups if they were particularly important. If we hadn't taken the time to go through this process, which was really time intensive then we wouldn't have succeeded in our aim. It would have been a much simpler process for us to roll out Manzama 'out of the box' but we wouldn't have cut through any of the 
existing noise in our current awareness service or provided any real value to our users.

\section{COMMUNICATIONS AND FINAL ROLL OUT}

We worked in partnership with our internal communications team and put together a comms plan to tell the various teams and groups involved in each stage of the rollout about the new service. Each user also received a welcome email which contained their login details and some links to bitesize videos showing how to further tailor their email.

We started the rollout process in June and had completed the practice area teams by October. We then focused on the remaining sector groups and our group services teams which were completed in April so the full rollout took us II months in total.

Throughout this stage we were actively monitoring the searches and sources to make sure that the content stayed relevant. We also added in additional sources and made changes on an ongoing basis based on feedback from our users. We set up a dedicated email address which was added onto the header of each daily email for users to contact us, along with links to a guide to passwords and access details for some of the sources included. We pulled all of the information together (top tips, bitesize videos, contact details etc) onto an intranet page which we linked to from our intranet homepage to further embed Manzama into the consciousness of our lawyers.

\section{FURTHER TRAINING}

Once the initial rollout was complete we felt we needed to give our users the skills to use the more advanced features of Manzama in order to allow them to further tailor their emails and use them to support their social media accounts. We did this in the New Year and travelled around the various Irwin Mitchell offices offering face-to-face training on Manzama. We sent out invitations on a team by team basis so we could also use the session as an opportunity to talk about existing pain points and how we could customise their updates to address some of these issues. On the back of these sessions we came away with lists of new searches to build and a real understanding of how users were using (or not) the updates and how we could make them more fit for purpose.

We also covered some of the basics again and encouraged people to unsubscribe from other email alerts, and highlighted specific things unique to Irwin Mitchell (like some of the bespoke searches), and some of the useful added features such as flagging articles, sharing content etc. This training proved really popular and we did notice an increase in people logging in to their profiles to amend and interact with the content from there rather than the email which was a real positive.

\section{WHAT HAVE WE ACHIEVED?}

We now have relevant content being pushed out regularly to over 1,700 people, mostly on a daily basis. Many people have unsubscribed from individual newsletters as the sources are included in their Manzama alerts which has helped to reduce email overload and gives those people one place to check for their news. However, it's worth mentioning that some people have chosen to keep some of their individual newsletters for various reasons but because we opted to set up Manzama on an individual rather than group profile basis this gives people the freedom to customise their own alert so that it does not include those sources they wish to subscribe to separately. In short, we have still reduced duplication whilst allowing people to get the information in the way that works for them.

We elected to go with individual profiles precisely because it gave people that freedom to tailor their alerts to their own interests and needs. However, as administrators the $\mathrm{K} \& \mathrm{l}$ team still have the ability to proactively push desired content out to people as well, which we do when we become aware through our own horizon scanning or from conversations within the business of something that a particular team or group of people within the business would need to monitor. We have also used that feature as an additional communication channel to push out news and messages from the K\&l team.

Metrics are always important in measuring return on investment so the fact we consistently have over 3,000 clicks a month and average over 400 unique users each month with a good spread of users across all areas of the business is encouraging but in some ways it's the anecdotal feedback that we get that is more important than the numbers. We've had really positive feedback from all areas of the business and all role levels including our CEO, who loves his alert and told our Partner \& Associate conference how great it was. We have heard first-hand examples of how something has been picked up in Manzama that would have a direct impact on our clients and as a result our fee-earners have been able to advise their clients accordingly.

There is also an efficiency factor to consider; not just in it being quicker for people to find news of interest to themselves but also making it quicker to share content with others both internally and externally. In particular, people who are responsible for the IM Twitter feeds are finding that instead of spending thirty minutes looking for stories to share this is now a five minute job. The automation itself has also freed up approximately a day a week of $\mathrm{K} \& \mathrm{l}$ team which can be used to focus on other projects.

\section{LESSONS LEARNT}

The soft launch and phased roll out worked really well. Not only did it give us time to make the content as good as possible from the start but we also found it created a 
bit of a buzz and generated its own momentum through word-of-mouth where we were getting people from teams in the latter phases asking us about it and wanting it for their teams.

The face-to-face training that we did post-launch was really well received and we have and will continue to offer that. If we were doing a similar project again we would probably do the face-to-face training a little earlier in the process and closer to the launch dates for each team. That would also have helped with getting the message out about Manzama generally to support the launch.

It's crucial to have people from within your business involved in creating the content and the roll out generally. As mentioned above the content needs to be relevant as soon as it goes out otherwise you lose people before you even start. Having that knowledge of your business and what your teams are interested in and not interested in really helps with that. We had an intensive five or six months where we focused on creation and testing of searches but that internal know how was invaluable and we feel it was worth the effort in getting it right. That does mean you have to take the time to really learn how to make the most of the system. We've definitely found that we got better at setting up searches as the roll out progressed and we picked up more tips and tricks so with hindsight we might have taken a little longer at the beginning with some test searches before we launched into it.

\section{NEXT STEPS}

We're very much into the business-as-usual phase with Manzama now which involves monitoring and reviewing existing searches, assisting with setting up new searches as required and training.

Manzama have also launched a new 'firm library' function, inspired at least in part, by the way we setup Manzama and the work they did with us to support that. This helps make it easier for users to find searches we have created in-house by browsing through a list rather than having to know what terms to search for. We've recently implemented that functionality and are promoting that to users as an improvement to how they can customise their alerts.

We've started looking at the possibilities around client-facing content including newsletters and pushing content out via client portals or extranets so we can offer this as a service. We're working on a couple of test cases at the moment and we're also looking to get our business development teams more involved to utilise some additional functionality that might be of interest to them.

\section{CONCLUSION}

Overhauling current awareness at Irwin Mitchell was a massive project for such a small team and it was not without its challenges, not least of which was managing other priorities around this and still sticking to our deadlines. However, it was also massively rewarding both in terms of the actual work and the feedback we have received from the business since its launch, which we think demonstrate we did achieve our ultimate aim of 'cutting through the noise'.

If anyone is considering a similar project I hope our experiences help you and if anyone is in the midst of a similar project then stick with it, it will be worth it in the end!

\section{Biographies}

Jill Henderson is a Knowledge \& Intelligence Advisor at Irwin Mitchell Solicitors. Since graduating from the University of Sheffield in 2010 with a first class honours BSc in Information Management she has been part of the Knowledge team at Irwin Mitchell and has been in her current role supporting the Business Legal Services teams with their knowledge and information needs for just under four years.

Louise Young is currently the Knowledge \& Intelligence Advisor for the Personal Legal Services division at Irwin Mitchell and has worked at IM since $200 \mathrm{I}$ in a variety of different roles. In the past she has specialised in delivering specialised legal and business research services to the business but in her current role she works with key contacts in the PLS practice teams to develop services and identify areas where KM can more effectively support the business. 